\title{
Islanding Detection for Microgrid Based on Frequency Tracking Using Extended Kalman Filter Algorithm
}

\author{
Bin Li, Jingpeng Wang, Hailong Bao, and Huiying Zhang \\ Key Laboratory of Smart Grid of Ministry of Education, Tianjin University, Tianjin 300072, China \\ Correspondence should be addressed to Bin Li; binli@tju.edu.cn
}

Received 23 January 2014; Revised 31 March 2014; Accepted 12 April 2014; Published 18 May 2014

Academic Editor: Hongjie Jia

Copyright (C) 2014 Bin Li et al. This is an open access article distributed under the Creative Commons Attribution License, which permits unrestricted use, distribution, and reproduction in any medium, provided the original work is properly cited.

\begin{abstract}
Islanding detection is essential for secure and reliable operation of microgrids. Considering the relationship between the power generation and the load in microgrids, frequency may vary with time when islanding occurs. As a common approach, frequency measurement is widely used to detect islanding condition. In this paper, a novel frequency calculation algorithm based on extended Kalman filter was proposed to track dynamic frequency of the microgrid. Taylor series expansion was introduced to solve nonlinear state equations. In addition, a typical microgrid model was built using MATLAB/SIMULINK. Simulation results demonstrated that the proposed algorithm achieved great stability and strong robustness in of tracking dynamic frequency.
\end{abstract}

\section{Introduction}

Distributed generation (DG) using renewable energy sources such as solar energy, wind energy, and hydroenergy has received considerable attention due to environmental pollution and exhaustion of fossil fuel. Many utilities around the world have a significant penetration of DGs in their systems. As a promising developing trend, the concept and techniques of microgrids (MGs) are proposed to improve DG's utilization [1].

MG is a kind of regional electric power systems which include DGs and power loads. They have the ability to disconnect from or parallel with large electric power systems. MGs offer many potential benefits, such as improving the reliability of power supply by islanding operation during large electric power system outages, relieving overload problems by allowing a part of the power system to intentionally island, and so forth. However, there are also many issues to be solved before MGs become integral part of the utilities, such as how to achieve high power quality, efficiency, and safety. Especially, the most concerned problem is the islanding which refers to a condition that MG has an independent powering to a location even though the MG has been disconnected from the grid [2]. This condition can be dangerous to grid workers who may not realize that the load is still powered. It can also cause adverse effects such as low power quality, grid protection interference, and equipment damage. For these reasons, islanding condition must be detected immediately. Accordingly, islanding condition should be informed to operators and the control strategy must be switched for islanding operation.

The mainly used islanding detection techniques may be broadly classified as remote and local techniques. Local techniques can be divided into passive and active detection methods [3]. Remote islanding detection techniques are based on the communication between utilities and DGs. Supervisory control and data acquisition (SCADA) has been used to determine whether the distribution system is islanded or not. These methods are reliable but not economic to implement for small systems. As for the local techniques, the core of passive method is that some of the system parameters (voltage, frequency, etc.) change greatly with islanding but not much in normal running when connected with grid $[4,5]$. This character can be helpful in islanding detection by continuously monitoring the parameters of the system without bringing disturbance. However, this method would cause large nondetection zones because islanding cannot be detected under a perfect match of generations and loads in the island system. Active techniques directly interact with the power system operation by introducing 


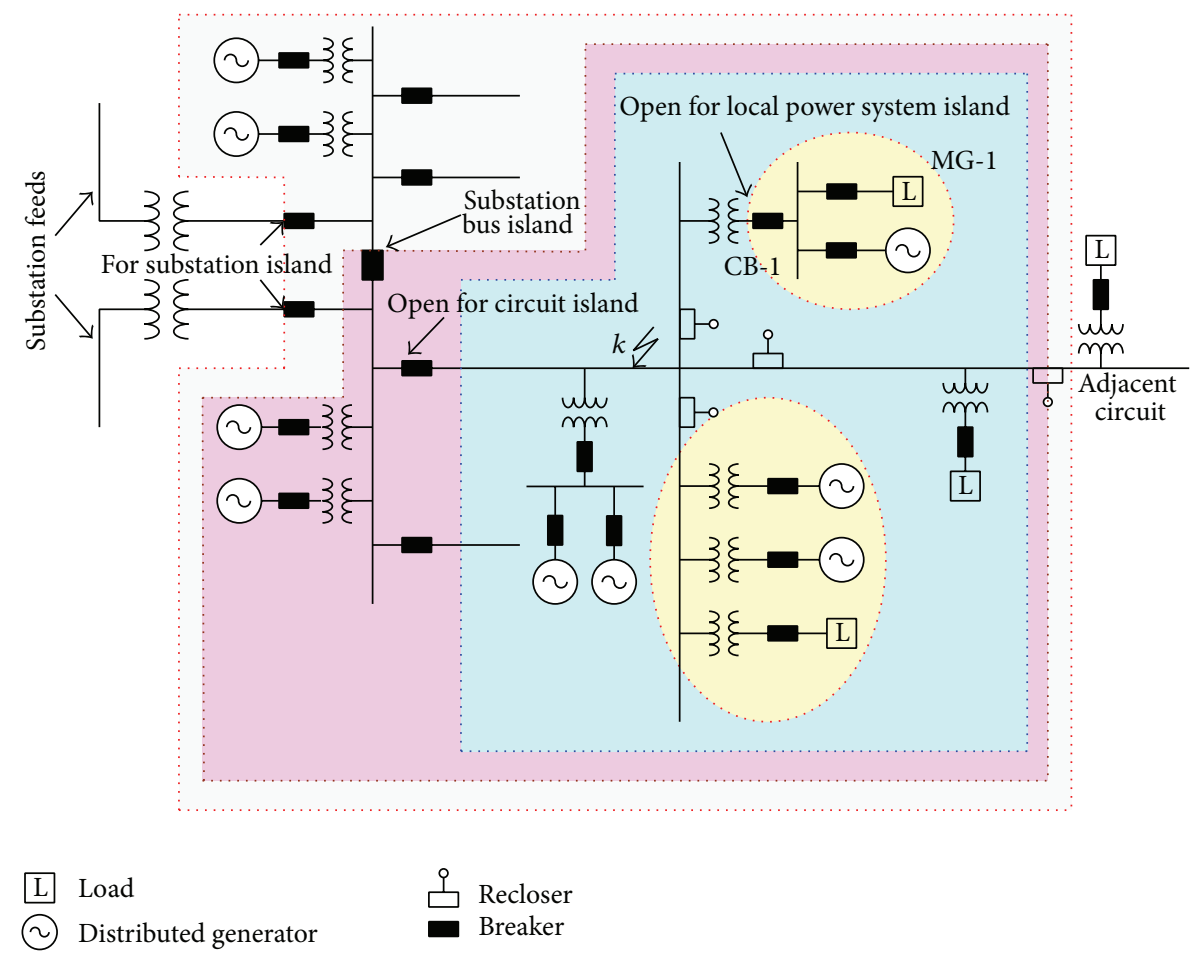

Figure 1: Typical topologies of MGs.

perturbations [6]. These small perturbations will result in a significant change in system parameters when the DG is islanded whereas the change will be negligible when the DG is connected to the grid. It has smaller nondetection zones but disturbs the system. The most commonly used methods are reactive power export error detection method, impedance measurement method, active frequency drift, and automatic phase shift.

For the passive detection method, one of the common passive approaches is based on the measurement of voltage vector and frequency of the common coupling point in real time. Variation in voltage and frequency from its normal value indicates the occurrence of islanding in most conditions. A large number of methods are available for the frequency estimation based on digitized samples of system voltage, methods such as discrete Fourier transforms, conventional Kalman filter, and phase lock loop [7]. For the most commonly used algorithms, discrete Fourier transforms may lead to inaccuracies due to leakage and picket-fence effects. Meanwhile, it is not ideal when the system is nonstationary since it requires large measurement windows and it is sensitive to low signal-to-noise ratio [8]. Kalman filter, which is well known for its small calculating amount and robustness in estimating sinusoids signal with noise, however, is faced with several distortions due to the system's inherent nonlinearity $[9,10]$.

In this paper, the necessity and basic methods of islanding detection of the MG are addressed. Considering the importance of frequency estimation, a novel algorithm based on extended Kalman filter is presented to enhance the precision and the speed of frequency tracking in islanding MGs.
Simulation tests demonstrate that the proposed algorithm has excellent superiority on islanding detection.

\section{Microgrids and Islanding Detection}

The MG embedded in distribution systems has evolved overwhelmingly to help meet the load growth in existing networks. The typical topologies of MGs are suggested in IEEE 1547.4 [11], as shown in Figure 1.

The most important feature of MGs is that they have the ability to disconnect from or parallel with the area electric power system. The planned MG in Figure 1 may be a local electric power system island, circuit island, or substation island. Generally, a transition-to-island mode can be a result of scheduled or unscheduled events. Scheduled transitions are intentional events for which the time and duration of the planned island are agreed upon by all parties involved. Unscheduled transitions are inadvertent events that are typically initiated by loss of area power system or equipment failure, and the MG needs to be automatically sectionalized from the area power system by islanding protective relay.

As shown in Figure 1, for a fault $k$ external to the planned island MG-1, it is expected that the DG should be disconnected from the area electric power system as soon as possible. Thus, the fast circuit breaker CB-1 is required to be open for the fault $k$. Obviously, knowledge of the operating conditions of the system prior to islanding and control of those operating conditions will facilitate the smooth transfer to an intentional island, particularly in response to abnormal events [12]. In order to achieve maximum benefit of the DG, the islanding relay should detect islanding condition with 


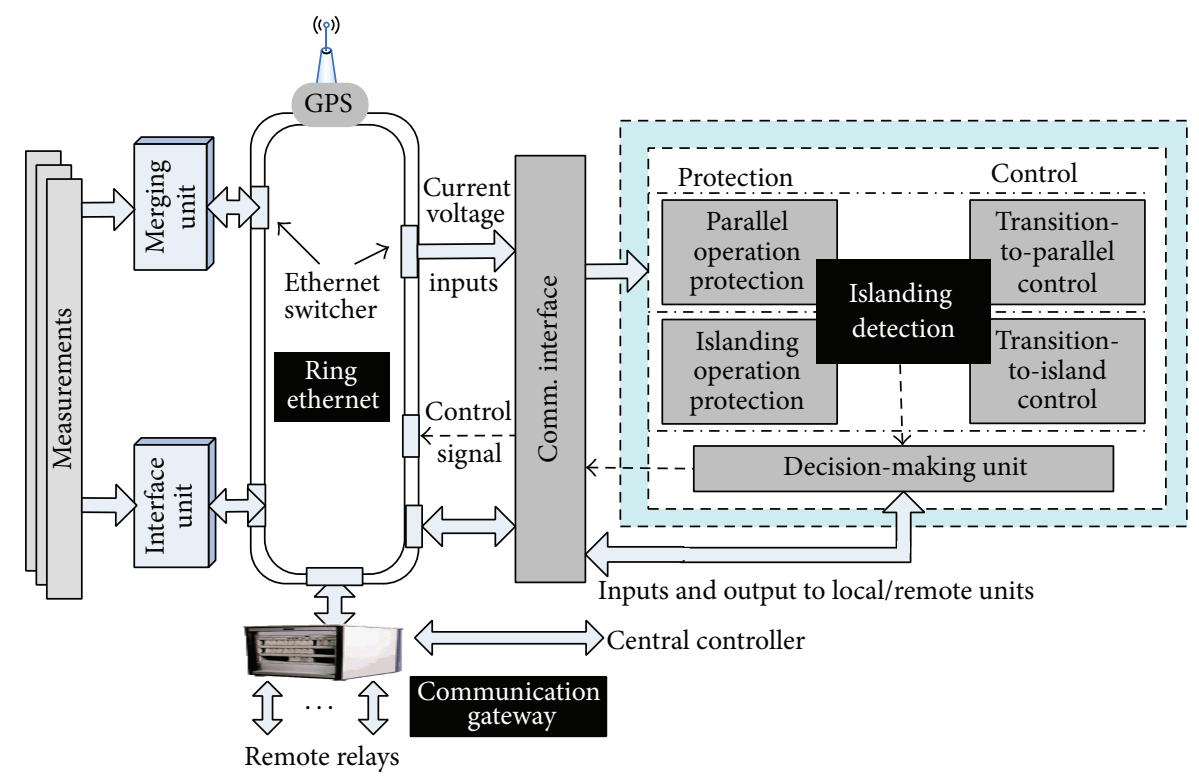

FIGURE 2: Schematic diagram of protection and control of MGs.

high speed and sensitivity to prevent other protective relays from tripping-off islanded DG.

In short, when islanding condition happens, not only the area electric power system operator needs to be aware of this operation, but also the control strategy of the MG needs to be adjusted. Therefore, islanding detection is one of the key issues for protection and control of MGs, as shown in Figure 2.

There are basically two kinds of distributed generators in MGs. The first one, such as photovoltaic, fan, and storage battery, is based on inverter control. The other one, such as synchronous generator and microsteam turbine, is based on the principle of generator.

Assume that the active and reactive power outputs from DGs are $P$ and $Q$ and that the power loads in MGs are represented as $R, L$, and $C$. In islanding operation,

$$
\begin{gathered}
P=\frac{U_{\mathrm{PCC}}^{2}}{R} \\
Q=U_{\mathrm{PCC}}^{2}\left(\frac{1}{\omega L-\omega C}\right) .
\end{gathered}
$$

So

$$
\begin{gathered}
U_{\mathrm{PCC}}^{2}=P R, \\
\omega=\omega_{0}\left(\sqrt{\frac{1}{4 \lambda^{2}}\left(\frac{Q}{P}\right)^{2}}+1-\frac{1}{2 \lambda} \cdot \frac{Q}{P}\right) .
\end{gathered}
$$

In (2), $\omega_{0}=1 / \sqrt{L C}$ is the syntonic angular frequency, and $\lambda=R \sqrt{C / L}$ is the quality factor of loads.

It can be known from (2) that the voltage and frequency may deviate from the rated ones when islanding occurs since there is generally an imbalance between the generation and the load in the formed island. Besides, the change of voltage is related to active power imbalance while the frequency shift is mainly based on reactive power imbalance. Consequently, over/undervoltage relay and over/underfrequency relay are often used as the most conventional anti-islanding protection. Furthermore, power imbalance will lead the frequency to change dynamically. So the rate of change of frequency (ROCOF) is also a commonly employed antiislanding protection [13]. Consider

$$
\frac{d f}{d t}=-\left(\frac{P_{L}-P_{G}}{2 H \times S_{\mathrm{GN}}} \times f_{r}\right) .
$$

Equation (3) is used to calculate the change rate of frequency, in which $P_{L}$ is the output of the distributed generator, $P_{G}$ is the load in the island, $S_{\mathrm{GN}}$ is the distributed generator rating, $H$ is the inertia constant of generating plant, and $f_{r}$ is the rated frequency.

The distributed generators in MGs are mainly based on inverter control. Compared with conventional synchronous generators, the inverter-based distributed generators have small equivalent inertia constants and capacities. The frequency varies distinctly in islanding operation. Furthermore, inverter control may produce more harmonics and noises due to dead time of the inverter itself. In sum, the voltage and the frequency of MGs are comparatively stable in parallel operation, while in islanding operation, the variations of electric measurements are complex, so that the accurate frequency tracking must be achieved by reliable and advanced algorithms.

\section{Frequency Tracking Using Extended Kalman Filter}

Frequency tracking could not be limited in islanding detection. It is needed in many other areas of power system, such as power quality monitoring, automation under frequency load 


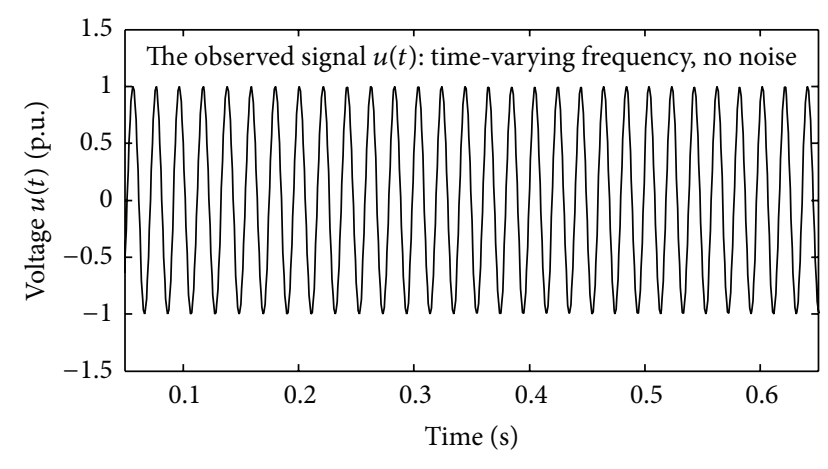

FIGURE 3: Voltage of observed signal with varying frequency.

shedding devices, and automatic accurate synchronizers. In theory, the frequency tracking methods can be investigated in all the situations that need frequency measurement.

A number of methods are available on the frequency estimation based on digitized samples of the system voltage. Discrete Fourier transform is one of the most commonly used algorithms due to its good filtering characteristics and strong resistance to disturbance. The main principle of discrete Fourier transforms is to calculate the phase angle difference between two adjacent data windows. Then the frequency can be estimated accordingly. However, discrete Fourier transforms may lead to inaccuracies due to leakage and picket-fence effects. Meanwhile, it is not ideal when the frequency is changing during a complete data window of Fourier transforms. For this reason, adaptive discrete Fourier transforms for frequency tracking are proposed to lock the fundamental frequency of incoming signal [14]. Adaptive discrete Fourier transforms can be described as a feedback model: when the signal frequency is changed, it can be used to redirect the sampling time of next sampling point. Thus, it enhances the accuracy of frequency measurements when the real frequency is not the rated value.

Kalman filter algorithm does well in addressing the general problem of trying to estimate the state $X \in R^{n}$ of a discrete-time controlled process. The superiority of Kalman filter in frequency tracking lies in many aspects.

(1) It is quite easy to make the Kalman filter self-tuned to the fundamental frequency without any hardware modification.

(2) Harmonics are eliminated more effectively when the Kalman filter is adaptive with respect to the fundamental frequency.

(3) The Kalman filter allows filtering preselected resonant nonharmonic frequencies, which is not possible with the adaptive discrete Fourier transforms.

(4) Kalman filter is often adopted due to its low computation cost and robustness in estimating sinusoids signal embedded in an unknown measurement noise [15].

However, the calculation of conventional Kalman filter is based on the assumption that the measured system is linear in its mathematical model. Thus, it has to face several distortions for coping with the nonlinear signals. As for the frequency tracking of power system, the input signal is sampling voltage values generally. If the input signal is assumed to be an ideal sinusoid with constant amplitude, the mathematical model can be represented as

$$
z(t)=A \cos \left(\omega_{0} t+\theta\right)=\left[\cos \left(\omega_{0} t\right)-\sin \left(\omega_{0} t\right)\right]\left[\begin{array}{l}
X_{1}(t) \\
X_{2}(t)
\end{array}\right\rceil,
$$

where $z(t)$ is the measured signal and $X_{1}(t)$ and $X_{2}(t)$ are the two state variables of Kalman filter, $\left[X_{1}(t), X_{2}(t)\right]^{T}=$ $[A \cos \theta, A \sin \theta]^{T}$.

Conventional Kalman filter supposes that $X_{1}(t)$ and $X_{2}(t)$ vary with time in linear way. However, as the mathematical model shows, the input signal and output signal are changed nonlinearly. It is hard for the conventional Kalman filter to achieve excellent calculation accuracy. With the interconnection of MGs, the system frequency is not as stable as the large power grid. When islanding occurs, the frequency of the MG might be irregular. Therefore, extended Kalman filter which is established in nonlinear way is recommended to be applied in order to achieve better accuracy and faster speed in frequency tracking of MGs.

The nonlinear stochastic difference equation can be written as

$$
\begin{gathered}
X_{k}=f\left(X_{k-1}, U_{k-1}, w_{k-1}\right), \\
z_{k}=h\left(X_{k}, v_{k}\right),
\end{gathered}
$$

where $X$ is state variables, $z$ is the observed signal, $U$ is the controlled variable, and subscript $k$ is the sampling time stamp. The nonlinear function $f$ in (5) describes nonlinear recursion relationship among state variables. The nonlinear function $h$ in (6) relates the state $X$ to the observed signal. $w$ and $v$ represent the process and measurement noise, respectively. It is assumed that $w(t)$ and $v(t)$ are both zeromean uncorrected white noises, with respect to variances $q$ and $r$. Consider $w(t)=W N(0, q), v(t)=V N(0, r)$.

To calculate state variables $X_{k}$ in nonlinear system, Taylor series is used to expand (5) and (6) and transform them into approximate linear equations $[16,17]$. For an input signal $f(x)$, it can be represented by

$$
\begin{aligned}
f(x)= & f(a)+\frac{f^{\prime}(a)}{1 !}(x-a) \\
& +\frac{f^{\prime \prime}(a)}{2 !}(x-a)^{2}+\cdots+\frac{f^{(n)}(a)}{n !}(x-a)^{n}+R n(x) .
\end{aligned}
$$

Generally, two-order Taylor series is adopted as an approximation solution in field calculation. This approximation is established based on three considerations: (1) higherorder components are so marginal that they can be omitted; (2) calculation of higher orders of Taylor series will result in heavy computing burden which influence the tracking speed; (3) the bigger Jacobian matrix is, the more memory is used, and it would be unsuitable in field application. 


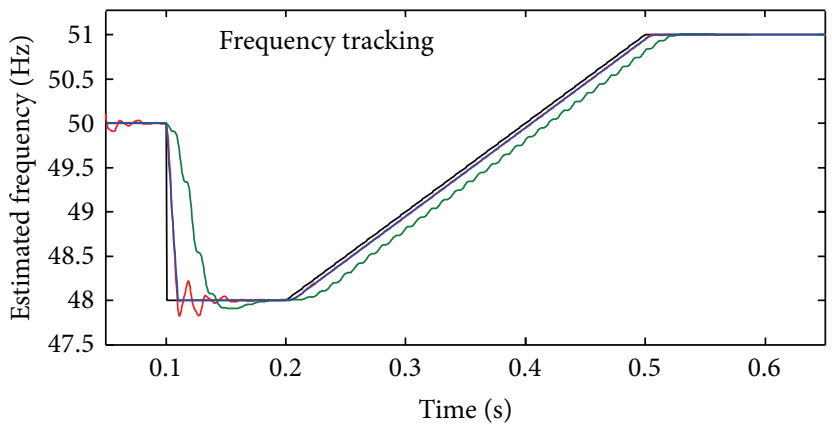

(a)

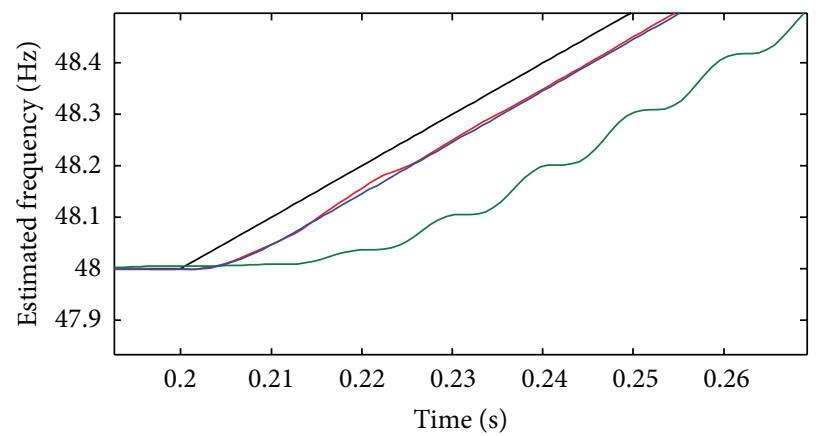

$\begin{array}{ll}\text { — Real frequency } & \text { Adaptive Fourier filter } \\ \text { Conventional Kalman filter } \quad \text { Extended Kalman filter }\end{array}$

(c)

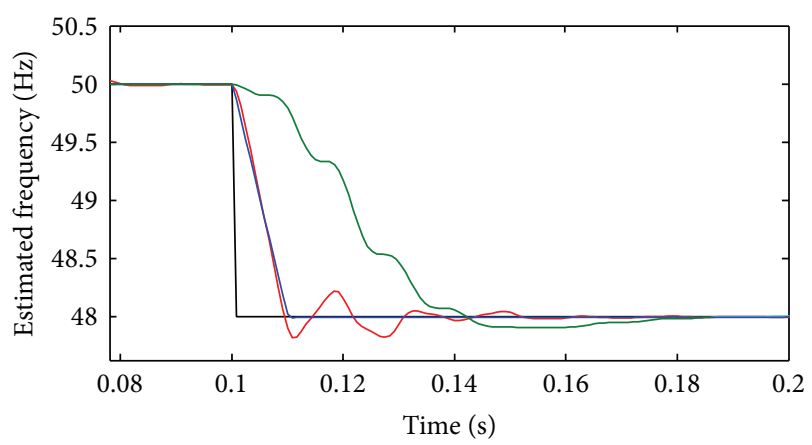

(b)

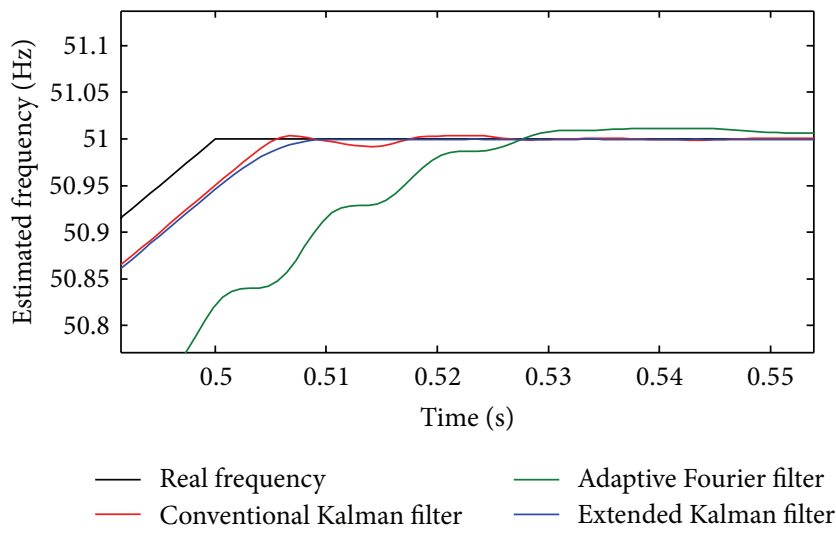

(d)

FIGURE 4: Frequency tracking performance. (a) Frequency tracking comparison among different algorithms; (b) measured frequencies in stage 1 ; (c) measured frequencies in stage 2; (d) measured frequencies in stage 3.

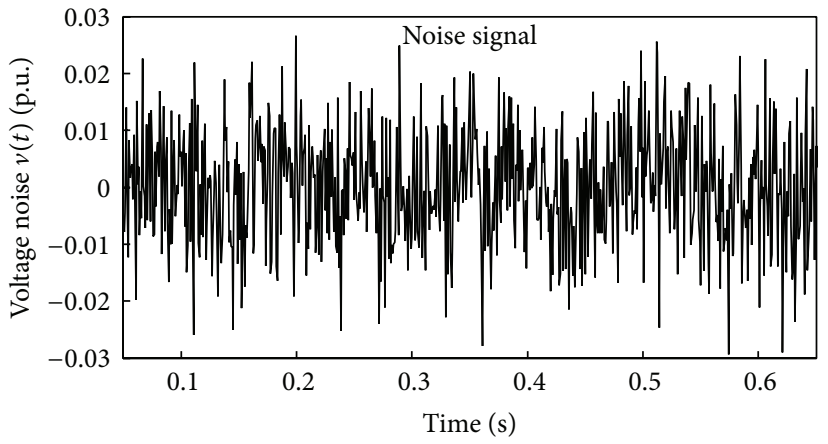

(a)

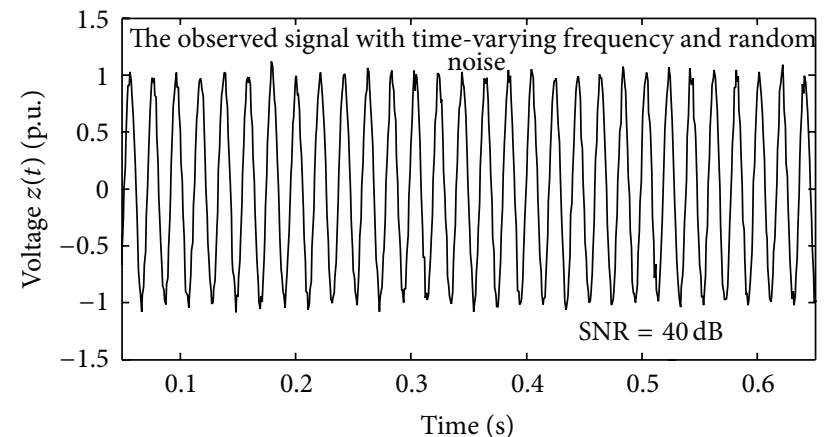

(b)

FIGURE 5: Observed signal with noise and time-varying frequency. (a) Noise signal; (b) the observed signal with time-varying frequency and random noise.

By using two-order Taylor series (7), (5), and (6) can be rewritten as

$$
\begin{gathered}
X_{k}=\widetilde{X}_{k}+F\left(X_{k-1}-\widehat{X}_{k-1}\right)+W \cdot w_{k-1}, \\
z_{k}=\widetilde{z}_{k}+H\left(X_{k}-\widetilde{X}_{k}\right)+V \cdot v_{k},
\end{gathered}
$$

where $X_{k}$ and $z_{k}$ represent the values of $X$ and $z$ in step $k$, respectively, and $\widetilde{X}_{k}$ and $\widetilde{z}_{k}$ represent the approximate values of $X$ and $z$ in Taylor series expansion. $\widehat{X}_{k-1}$ represents the posterior estimation value of the state variable in step $k-1 . H$ is called measurement matrix. $W$ and $V$ are Jacobian matrixes of $w$ and $v$, respectively. $F$ is the Jacobian matrix of $f$ and represents the partial derivatives on $X$. Consider

$$
\begin{gathered}
W_{k}=\frac{\partial f(x)}{\partial w}\left(\widehat{X}_{k-1}, u_{k-1}, w_{k-1}\right), \\
V_{k}=\frac{\partial h(x)}{\partial v}\left(\widetilde{X}_{k}, v_{k}\right),
\end{gathered}
$$




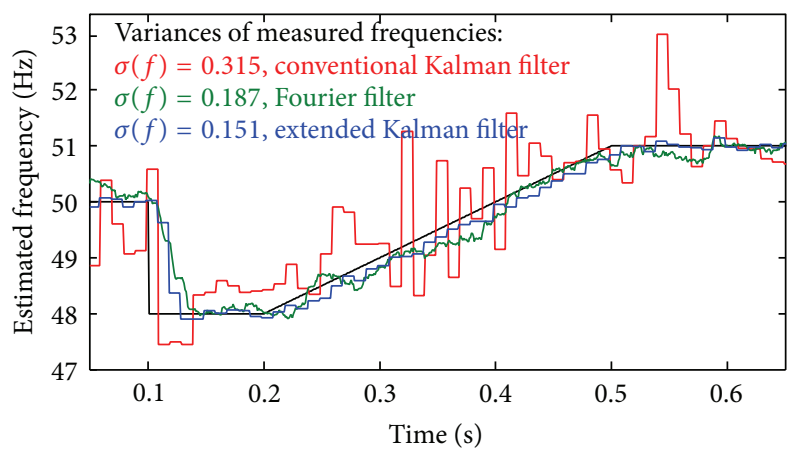

- Real frequency $\quad$ Adaptive Fourier filter

FIGURE 6: Frequency tracking performance for the signal with noise and time-varying frequency.

$$
F(k)=\left.\frac{\partial f(x)}{\partial x}\right|_{x=\widehat{X}(k-1)}
$$

For the extended Kalman filter, $F$ is not a constant matrix during iteration calculation since $f$ is a nonlinear function. It is one of the main differences between conventional Kalman filter and extended Kalman filter.

The initial stage of extended Kalman filter design is to model the signal and derivation of state variables of it. This is because the signal model dynamics describe a mechanism for how the process may evolve. Considering the electric measurements (voltages, currents, and frequencies) in MGs may change irregularly, or nonlinearly in most cases, Taylor series is used to nonlinear calculation. The voltage at the point of common coupling in MGs can be described approximately as follows:

$$
z(t)=A(t) \cos (\omega(t) \cdot t+\varphi)
$$

where $A(t)$ and $\varphi$ represent the amplitudes and initial phase.
Using the cosine expansion, we can obtain the discrete expression of the following:

$$
\begin{gathered}
z\left(k T_{s}\right)=A\left(k T_{s}\right) \cos \left[\omega\left(k T_{s}\right) \cdot(k-1+1) T_{s}+\varphi\right] \\
=A\left(k T_{s}\right)\left\{\cos \left[\omega\left(k T_{s}\right) \cdot(k-1) T_{s}+\varphi\right] \cdot \cos \omega\left(k T_{s}\right)\right. \\
-\sin \left[\omega\left(k T_{s}\right) \cdot(k-1) T_{s}+\varphi\right] \\
\left.\cdot \sin \omega\left(k T_{s}\right)\right\} .
\end{gathered}
$$

The amplitude and frequency of voltage at the point of common coupling are changing when islanding occurs. Therefore,

$$
\begin{gathered}
\omega\left(k T_{s}\right)=(1-\varepsilon) \cdot \omega\left[(k-1) T_{s}\right], \\
A\left(k T_{s}\right)=\alpha_{k-1} \cdot A\left[(k-1) T_{s}\right] .
\end{gathered}
$$

In (12), $\varepsilon$ represents the rate of frequency variation. Since the frequency variation between two sampling steps is very small, $0<\varepsilon \ll 1 . \alpha_{k-1}$ is the coefficient of amplitude variation and it can be considered as a new state variable in mathematical modeling. Accordingly, (11) can be rewritten as

$$
\begin{aligned}
z\left(k T_{s}\right)=\alpha_{k-1} & \times A\left[(k-1) T_{s}\right] \\
\times\left\{\cos \left\{\omega\left[(k-1) T_{s}\right] \cdot(1-\varepsilon) \cdot(k-1) T_{s}+\varphi\right\}\right. & \\
\cdot & \cos \left\{\omega\left[(k-1) T_{s}\right] \cdot(1-\varepsilon)\right\} \\
& -\sin \left\{\omega\left[(k-1) T_{s}\right] \cdot(1-\varepsilon) \cdot(k-1) T_{s}+\varphi\right\} \\
& \left.\cdot \sin \left\{\omega\left[(k-1) T_{s}\right] \cdot(1-\varepsilon)\right\}\right\} .
\end{aligned}
$$

In this paper, four state variables are defined as in-phase signal, quadrature signal, time-varying frequency, and timevarying amplitude, which are all changing in nonlinear way as proceeded.

Consider

$$
\begin{aligned}
X(k) & =\left[\begin{array}{l}
X_{1}(k) \\
X_{2}(k) \\
X_{3}(k) \\
X_{4}(k)
\end{array}\right]=\left[\begin{array}{c}
A\left(k T_{s}\right) \cos \left\{\omega\left(k T_{s}\right) \cdot k T_{s}+\varphi\right\} \\
A\left(k T_{s}\right) \sin \left\{\omega\left(k T_{s}\right) \cdot k T_{s}+\varphi\right\} \\
\omega\left(k T_{s}\right) \\
\alpha_{k} \cdot A\left(k T_{s}\right)
\end{array}\right] \\
= & {\left[\begin{array}{c}
X_{4}(k-1) \cdot\left[\cos \left(X_{3}(k-1)\right) \cdot X_{1}(k-1)-\sin \left(X_{3}(k-1)\right) \cdot X_{2}(k-1)\right] \\
X_{4}(k-1) \cdot\left[\sin \left(X_{3}(k-1)\right) \cdot X_{1}(k-1)+\cos \left(X_{3}(k-1)\right) \cdot X_{2}(k-1)\right] \\
(1-\varepsilon) \cdot X_{3}(k-1)+w\left(k T_{s}\right) \\
X_{4}(k-1)
\end{array}\right] . }
\end{aligned}
$$

Based on the relationship of $X_{k}$ and $X_{k-1}$, we can get the expression of nonlinear function $f\left(X_{k}\right)$ and the Jacobian matrix $F\left(X_{k}\right)$. Consequently, the detailed calculation steps are as follows.
(1) Predict the state with initials:

$$
\widehat{X}_{k}^{-}=f\left(\widehat{X}_{k-1}^{-}, w_{k-1}\right)
$$




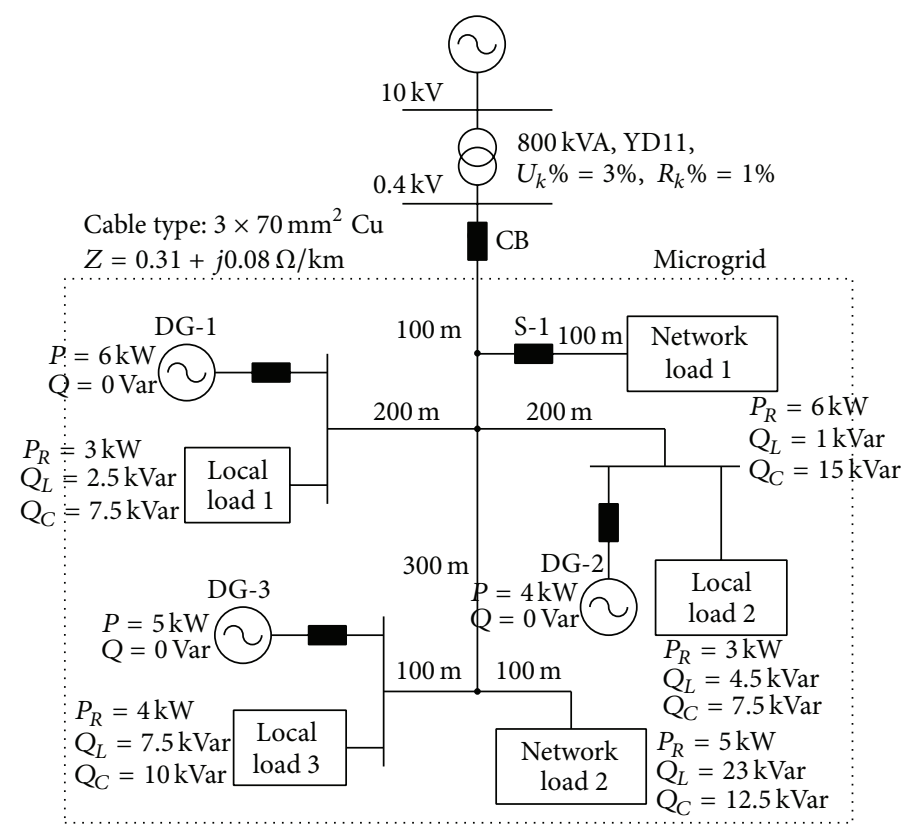

FIGURE 7: Simulation model of distribution network with MGs.

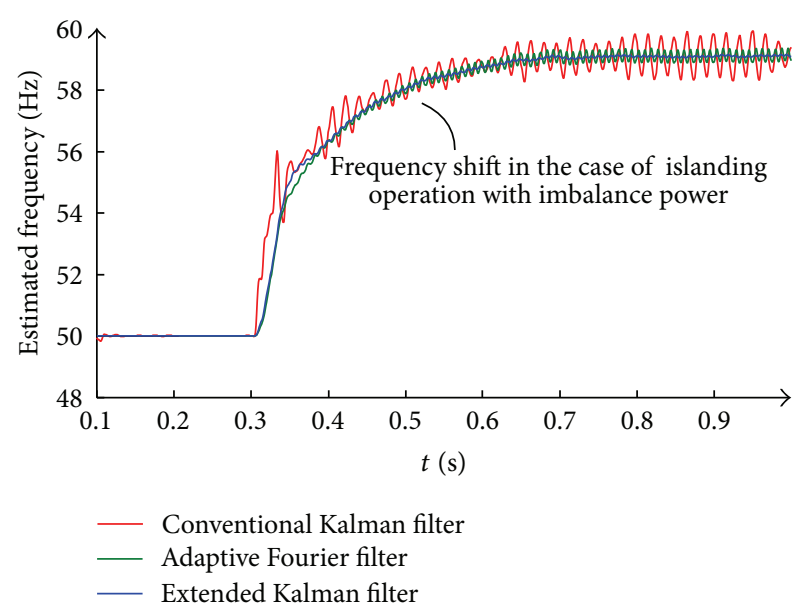

FIGURE 8: Frequency shift when islanding occurs with imbalanced power.

(2) Compute the error covariance:

$$
P_{k}^{-}=F_{k} P_{k-1} F_{k}^{T}+W_{k} Q_{k-1} W_{k}^{T} .
$$

(3) Compute the Kalman gain:

$$
K_{k}=P_{k} H_{k}^{T} \cdot\left(H_{k} P_{k}^{-} H_{k}^{T}+V_{k} R_{k} V_{k}^{T}\right) .
$$

(4) Update the state estimate:

$$
\widehat{X}_{k}=\widehat{X}_{k}^{-}+K_{k} \cdot\left(z_{k}-h\left(\widehat{X}_{k}^{-}, 0\right)\right) .
$$

(5) Update the error covariance:

$$
P=\left(I-K_{k} H_{k}\right) \cdot P_{k}^{-} .
$$

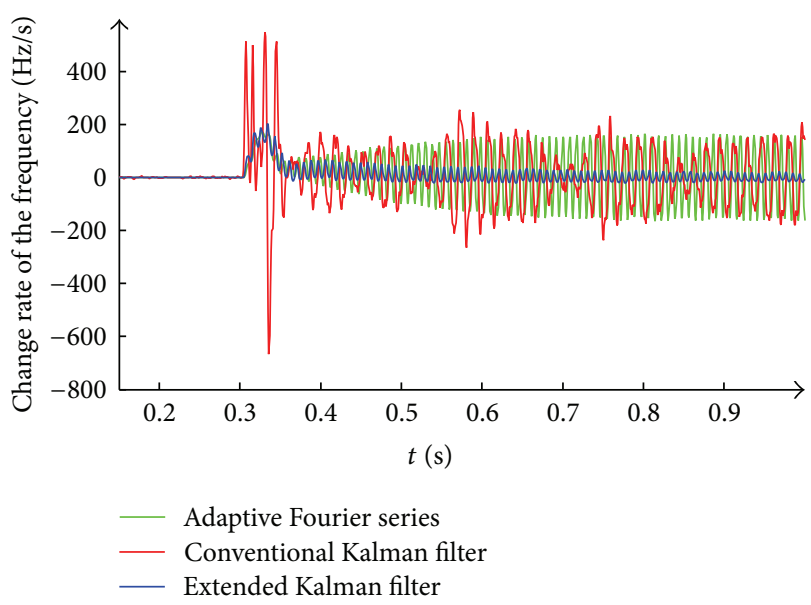

FIGURE 9: Change rate of the frequency when islanding occurs with imbalanced power.

\section{Case Studies}

Compared with the other two common frequency tracking methods, adaptive discrete Fourier transforms (i.e., adaptive Fourier filter) and conventional Kalman filter, the performance of extended Kalman filter-based frequency calculation algorithm is experimentally evaluated. For the following simulation tests, the fundamental frequency and amplitude are assumed to be $50 \mathrm{~Hz}$ and 1 p.u., respectively. The sampling frequency is set to be $1200 \mathrm{~Hz}$, which is the conventional choice for protective relays.

Firstly, the observed signal is a voltage waveform with time-varying frequency; that is, $u(t)=\sin (\omega(t) * t+\varphi)$. The angular frequency $\omega(t)$ changes from $50 \mathrm{~Hz}$ down to $48 \mathrm{~Hz}$ 


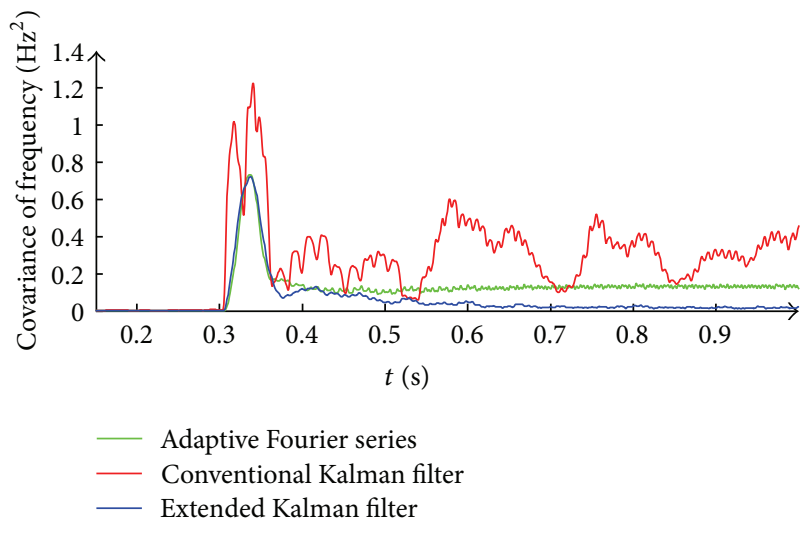

FIGURE 10: Frequency variance when islanding occurs with imbalanced power.

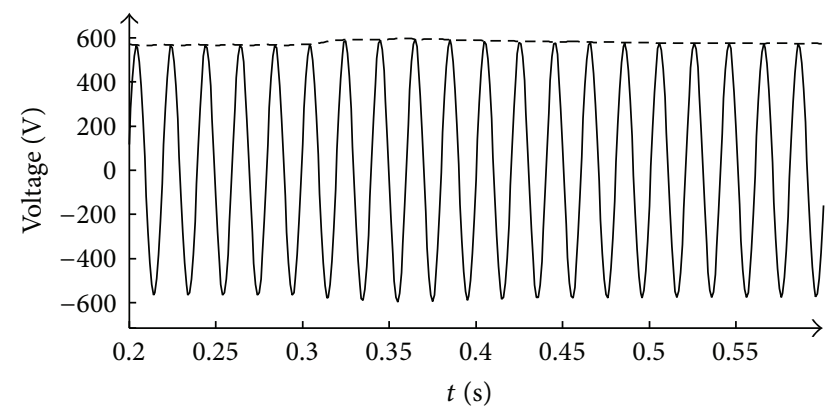

FIGURE 11: Measured voltage of islanding MG when islanding occurs with balanced power.

and then climbs to $51 \mathrm{~Hz}$ linearly. The actual voltage is shown in Figure 3.

Accordingly, frequency tracking performance of different algorithms is shown in Figure 4.

To explain clearly, the test can be divided into three stages.

(1) Stage 1 (2 Hz step-down change in frequency). The frequency of the signal is suddenly decreased from $50 \mathrm{~Hz}$ to $48 \mathrm{~Hz}$. The results reveal that the tracking time of extended Kalman filter is about $10 \mathrm{~ms}$, while the other two algorithms use almost $50 \mathrm{~ms}$. Extended Kalman filter is 4 times faster than the others in frequency tracking.

(2) Stage 2 (tracking the ramp). The proposed extended Kalman filter-based algorithm has the less responding time in tracking frequency variation. As shown in Figure 4, extended Kalman filter and conventional Kalman filter both have good performance in tracking ramping frequency. Their tracking times in this case are $20 \mathrm{~ms}$ shorter than adaptive Fourier filter's.

(3) Stage 3 (back to $51 \mathrm{~Hz}$ ). Extended Kalman filter-based algorithm provides frequency estimation result with high accuracy and velocity. Still, extended Kalman filter and conventional Kalman filter are better than adaptive Fourier filter and extended Kalman algorithm has the best stability compared with these three methods.

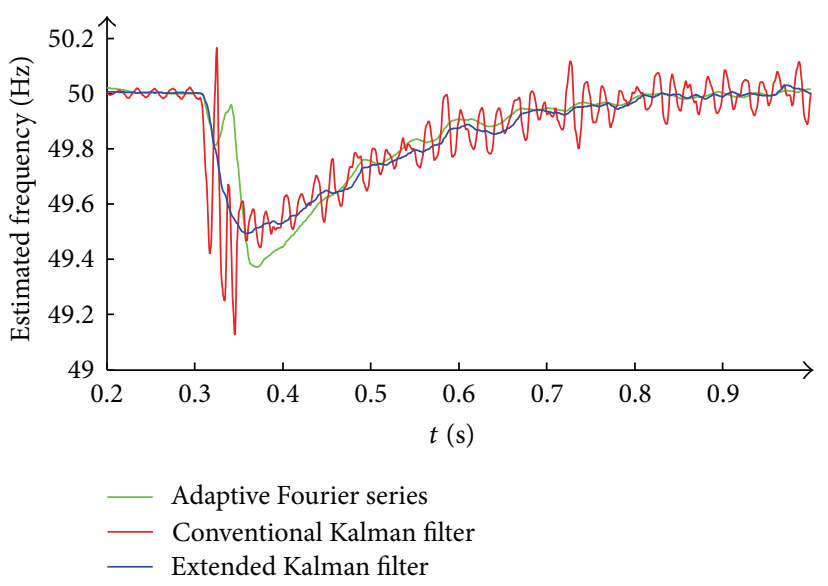

FIGURE 12: Frequency shift when islanding occurs with balanced power.

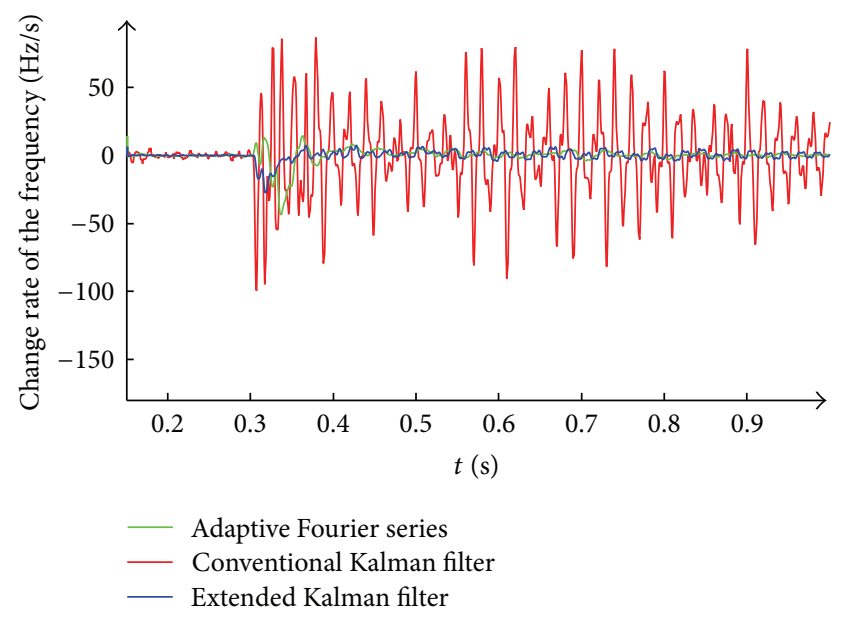

FIGURE 13: Change rate of the frequency when islanding occurs with balanced power.

For the tracking time of ramp-changed frequency, extended Kalman filter and conventional Kalman filter are almost the same. However, extended Kalman filter is more stable and has less fluctuation than the conventional one. Besides, as the frequency changes sharply, the proposed method has clear superiority in tracking speed. In sum, it is confirmed that the proposed extended Kalman filter-based algorithm has excellent responding performance for signals with time-varying frequencies.

In addition, it is necessary to study the response of the extended Kalman filter and the other two algorithms in the presence of noise. Random noise is injected to the observed signal to test the stability of the algorithms. Generally, the signal-to-noise ratio, often written as SNR, is the measure of signal strength relative to background noise:

$$
\mathrm{SNR}=20 \lg \left(\frac{V_{S}}{V_{N}}\right)
$$




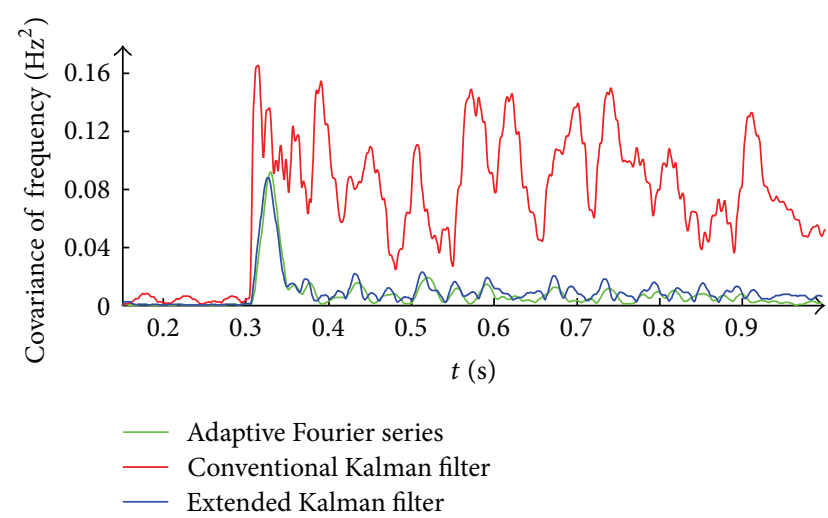

FIGURE 14: Frequency variance when islanding occurs with imbalanced power.

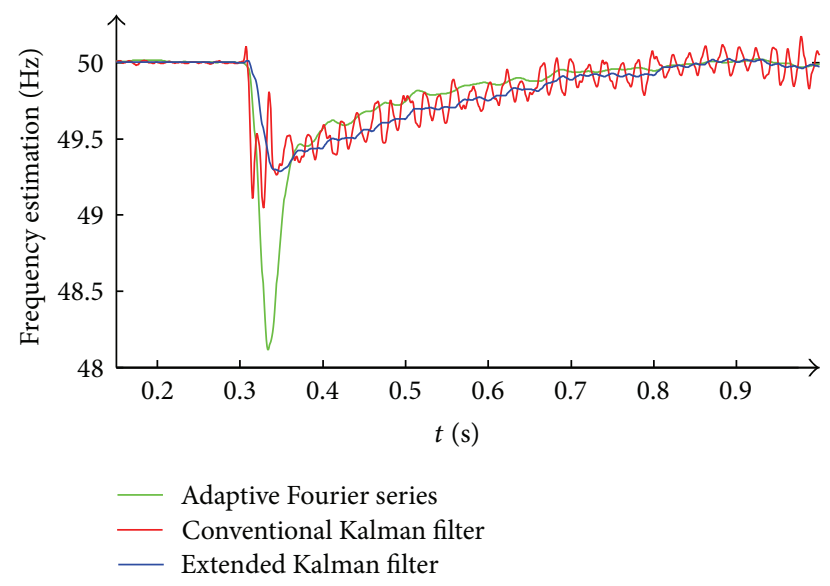

FIGURE 15: Frequency shift when islanding occurs with active power imbalance only.

In (21), $V_{S}$ is the observed signal strength in volts and $V_{N}$ is the noise level also in volts. The ratio is measured in decibels (dB).

Obviously, the observed signal is more unreadable with the larger noise. Even a little noise can cause a big error in frequency tracking. This is because such comprehensive signal is no longer ideal sine or cosine wave for noise added to pure wave. Based on the observed signal $u(t)$ shown in Figure 3, random noise $v(t)$ was added in $u(t)$ to form signal $z(t)$, in which SNR is set to be $40 \mathrm{~dB}$, as shown in Figures 5(a) and 5(b).

For the signal $z(t)$ with time-varying frequency and noise, it is hard to estimate frequency correctly. Figure 6 shows the estimated frequencies by Kalman filter, adaptive Fourier filter, and extended Kalman filter-based algorithms.

Since the linear characteristics of local signal were deteriorated by adding noise, conventional Kalman filter was influenced significantly. Apparently, the proposed algorithm based on extended Kalman filter and the algorithm based on adaptive Fourier filter achieved better performance in antinoise ability. For comparison, the measured frequency variances are 0.187 for adaptive Fourier filter-based algorithm and 0.151 for extended Kalman filter-based algorithm, respectively. Therefore, the proposed algorithm is more effective for frequency tracking under the conditions of islanding operation with time-varying frequency, noise, and so forth.

As shown in Figure 4, the proposed algorithm achieves best tracking performance for the input signal without noise. Adaptive Fourier algorithm has the worst frequency tracking character in this case. Figure 6 demonstrates that when there exists noise, adaptive Fourier filter obtains better antinoise ability than conventional Kalman filter. The proposed algorithm still achieves the best antinoise ability. These results show that (1) adaptive Fourier filter has a better antinoise performance but has the poorest frequency tracking ability, (2) conventional Kalman filter has a better frequency tracking performance but has the poorest antinoise ability, and (3) the proposed algorithm based on extended Kalman filter has the best performance in both frequency tracking and antinoise.

Generally speaking, the most commonly used method in islanding detection of MGs is both over/undervoltage relay and over/underfrequency relay (or ROCOF). Over/undervoltage relay mainly works when there is an active power imbalance, while over/underfrequency is more effective when there is a reactive power imbalance. Thus, frequency tracking ability of the proposed algorithm can be tested in a designed islanding simulation model.

By using MATLAB/SIMULINK, the model of distribution network with MGs is built to simulate various parallel and islanding operation conditions. As shown in Figure 7, the distribution network contains several DGs and energy storage components, transmission line, and load. The parameters of distribution network are listed in Figure 7. To verify the good performance of extended Kalman filter, different topologies of MGs have been formed to build a variety of operation conditions. In this paper, three typical islanding conditions are discussed as follows.

Case 1 (islanding operation with imbalanced power (both active and reactive)). The circuit breaker $\mathrm{CB}$ opens and the MG is in islanding operation. According to the output of DGs and amounts of power loads, there is an obvious imbalance between the generations and loads in the islanding MG. Based on (1) and (2), the frequency of MG-1 should have a sudden change consequently when the island happens. By applying these three algorithms in frequency tracking, results are shown in Figure 8.

As shown in Figure 8, when there are obvious imbalance between powers and loads in the MG, the frequency raised from fundamental frequency to $60 \mathrm{HZ}$ gradually. Obviously, in the process of the frequency growth, the stability of extended Kalman Filter-based algorithm is much better than the other two algorithms. In such cases, islanding with imbalanced power can be easily detected by over/underfrequency relay. The proposed frequency estimation algorithm can be embedded to improve calculation accuracy and stability with less fluctuation.

The measurements of frequency changing rate $(d f / d t)$ and frequency variance are shown in Figures 9 and 10.

As shown in Figure 9, there is a sudden change for all the three algorithms at about $0.3 \mathrm{~s}$ (exact islanding time) 


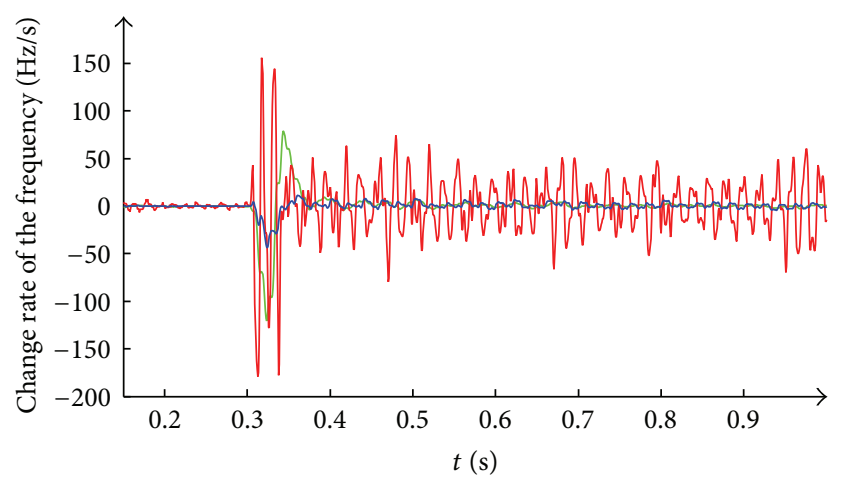

Adaptive Fourier series
_ Conventional Kalman filter
Extended Kalman filter

FIGURE 16: Change rate of the frequency when islanding occurs with active power imbalance only.

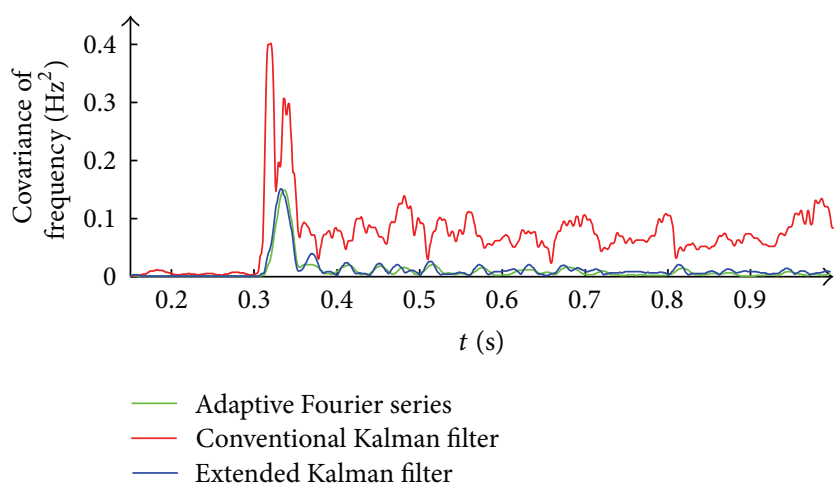

FIGURE 17: Frequency variance when islanding occurs with active power imbalance only.

in the simulation. This sudden change can also be used to exam whether there is an islanding or not. After $0.3 \mathrm{~s}, d f / d t$ is supposed to gradually change to zero and then stabilize. This is because there is no more power imbalance except for some small disturbance to cause frequency change. As for adaptive Fourier filter, $d f / d t$ remains obviously to fluctuate after $0.05 \mathrm{~s}$ of islanding process. $d f / d t$ fluctuates cyclically in conventional Kalman filter. Extended Kalman filter is found to be stable after islanding process, unlike earlier approaches.

Figure 10 shows the change tendency of frequency covariance when islanding occurs with imbalanced power. Frequency covariance reflects the difference between average value and sample value. In theory, frequency covariance should increase when there is an island and then decrease to zero gradually as the frequency stabilizes. It is obvious in Figure 10 that extended Kalman filter is the most stable one with the least fluctuation.

Simulation results show that extended Kalman filter not only can detect frequency changes effectively but also has less fluctuation after islanding process.

Case 2 (islanding operation with balanced power). In islanding detection, protection relay should act quickly when an island happens, regardless of whether the powers of DGs and local loads are matched or not.

In case 2, switch S-1 is disconnected; network load 1 is out of power consequently. The total numbers of generations are equal to the sum of loads for both active and reactive powers. The circuit breaker CB opens to form an MG at $0.3 \mathrm{~s}$. In theory, the voltage and frequency of the MG will change a little. The measured voltage and frequency are shown in Figures 11 and 12.

As shown in Figures 11 and 12, the measured voltage changes a little right after $0.3 \mathrm{~s}$; the frequency also changes to approximately $49.5 \mathrm{HZ}$ in the islanding process. It is also seen from the simulations that extended Kalman filter changes the fastest with the least fluctuations compared with the other two algorithms.

Figure 13 shows the performance of the three algorithms for detecting $d f / d t$ in the MG and it is observed from the figure that $d f / d t$ detected by conventional Kalman filter becomes divergent after islanding, while the other two algorithms can detect the small changes when islanding happens with balanced power. For extended Kalman filter, the tracking time is $0.01 \mathrm{~s}$ (approximately) less than adaptive Fourier filter. The variance of the three algorithms is shown in Figure 14.

Case 3 (islanding operation with only imbalanced active power). In case $3, \mathrm{DG}-1$ is changed to $3 \mathrm{~kW}$ and the switch $\mathrm{S}-1$ is kept disconnected. Consequently, there is only a $3 \mathrm{~kW}$ shortage of active power in the MG when islanding happens. In theory, voltage of PCC should change a lot but frequency would change a little because frequency shift in MG is mainly influenced by reactive power shortage. Figures 15, 16, and 17 show estimation values of frequency, $d f / d t$, and variance of frequency, respectively. Obviously, simulation results verify that the estimated frequency by using the proposed algorithm is more stable than those of the other two algorithms when islanding occurs.

\section{Conclusion}

This paper presents an extended Kalman filter for the frequency estimation to detect islanding in MGs. The mathematical model of nonlinear system is developed using analytical equations. In order to solve the nonlinear problem, Taylor series are used to linearize state equations, which is the main point of solving the nonlinear problem compared with other algorithms. To show the performance of extended Kalman filter, several tests have been applied. The simulation results show that extended Kalman filter not only has the fastest tracking speed compared with conventional Kalman filter and adaptive Fourier filter in test 1 but also has the best interference immunity in the presence of random noise in test 2. It can stand $40 \mathrm{~dB}$ or more noise without much distortion. By using extended Kalman filter, the islanding detection based on frequency is improved significantly in reliability and stability. 


\section{Conflict of Interests}

The authors declare that there is no conflict of interests regarding the publication of this paper.

\section{Acknowledgments}

This work was supported by the National High Technology Research and Development Program of China (2011AA05A106), Program for New Century Excellent Talents in Education Ministry of China (no. NCET-11-0367), and National Natural Science Foundation of Tianjin City (11JCYBJC07600).

\section{References}

[1] J.-H. Kim, J.-G. Kim, Y.-H. Ji, Y.-C. Jung, and C.-Y. Won, "An islanding detection method for a grid-connected system based on the goertzel algorithm," IEEE Transactions on Power Electronics, vol. 26, no. 4, pp. 1049-1055, 2011.

[2] P. Mahat, Z. Chen, and B. Bak-Jensen, "Review of islanding detection methods for distributed generation," in Proceedings of the 3rd International Conference on Deregulation and Restructuring and Power Technologies (DRPT '08), pp. 2743-2748, April 2008.

[3] S. P. Chowdhury, S. Chowdhury, C. F. Ten, and P. A. Crossley, "Islanding protection of distribution systems with distributed generators-a comprehensive survey report," in Proceedings of the IEEE Power and Energy Society General Meeting, pp. 1-8, July 2008.

[4] E. Kamyab and J. Sadeh, "Islanding detection method for photovoltaic distributed generation based on voltage drifting," IET, Generation, Transmission \& Distribution, vol. 7, no. 6, pp. 584-592, 2013.

[5] M. Yingram and S. Premrudeepreechacharn, "Investigation over/under-voltage protection of passive islanding detection method of distributed generations in electrical distribution systems," in Proceedings of the International Conference on Renewable Energy Research and Applications, pp. 1-5, Nagasaki, Japan, November 2012.

[6] D. D. Reigosa, F. Briz, C. B. Charro, and P. Garcia, "Active islanding detection using high-frequency signal injection," IEEE Transactions on Industry Applications, vol. 48, no. 5, pp. 1588-1597, 2012.

[7] P. K. Dash, A. K. Pradhan, and G. Panda, "Frequency estimation of distorted power system signals using extended complex Kalman filter," IEEE Transactions on Power Delivery, vol. 14, no. 3, pp. 761-766, 1999.

[8] A. G. Phadke, J. S. Thorp, and M. G. Adamiak, "A new measurement technique for tracking voltage phasors, local system frequency and rate of change of frequency," IEEE Transactions on Power Apparatus and Systems, vol. 102, no. 5, pp. 1025-1038, 1983.

[9] P. K. Ray and B. Subudhi, "Ensemble-Kalman-filter-based power system harmonic estimation," IEEE Transactions on Instrumentation and Measurement, vol. 61, no. 12, pp. 3216-3224, 2012.

[10] G. Valverde and V. Terzija, "Unscented Kalman filter for power system dynamic state estimation," IET Generation, Transmission and Distribution, vol. 5, no. 1, pp. 29-37, 2011.
[11] IEEE Std 1547.4-2011, "IEEE guide for design, operation, and integration of distributed resource island systems with electric power systems," in Proceedings of the IEEE Standards Coordinating Committee 21, pp. 1-54, July 2011.

[12] S. Kusagawa, E. Masada, J. Baba, M. Ohshima, and I. Nagy, "Coordinate control of distributed generations with power converters in a micro grid," in Proceedings of the European Conference on Power Electronics and Applications, pp. 1-10, Dresden, Germany, September 2005.

[13] C. F. Ten and P. A. Crossley, "Evaluation of ROCOF relay performances on networks with distributed generation," in Proceedings of the IET 9th International Conference on Developments in Power Systems Protection (DPSP '08), pp. 522-527, Glasgow, UK, March 2008.

[14] G. Benmouyal, "Adaptive sampling-interval generator for digital relaying," IEEE Transactions on Power Delivery, vol. 4, no. 3, pp. 1602-1609, 1989.

[15] A. A. Girgis and T. L. D. Hwang, "Optimal estimation of voltage phasors and frequency deviation using linear and non-linear Kalman filtering: theory and limitations," IEEE Transactions on Power Apparatus and Systems, vol. 103, no. 10, pp. 2943-2951, 1984.

[16] B.F. la Scala and R.R. Bitmead, "Design of an extended Kalman filter frequency tracker," in Proceedings of the IEEE International Conference on Acoustics, Speech, and Signal Processing, pp. 525527, Adelaide, Australia, April 1994.

[17] C.-H. Huang, C.-H. Lee, K.-J. Shih, and Y.-J. Wang, "Frequency estimation of distorted power system signals using a robust algorithm," IEEE Transactions on Power Delivery, vol. 23, no. 1, pp. 41-51, 2008. 


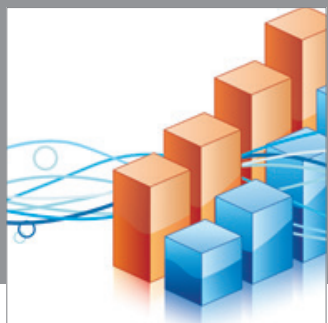

Advances in

Operations Research

mansans

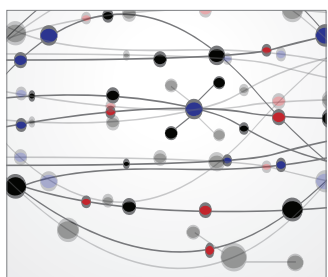

The Scientific World Journal
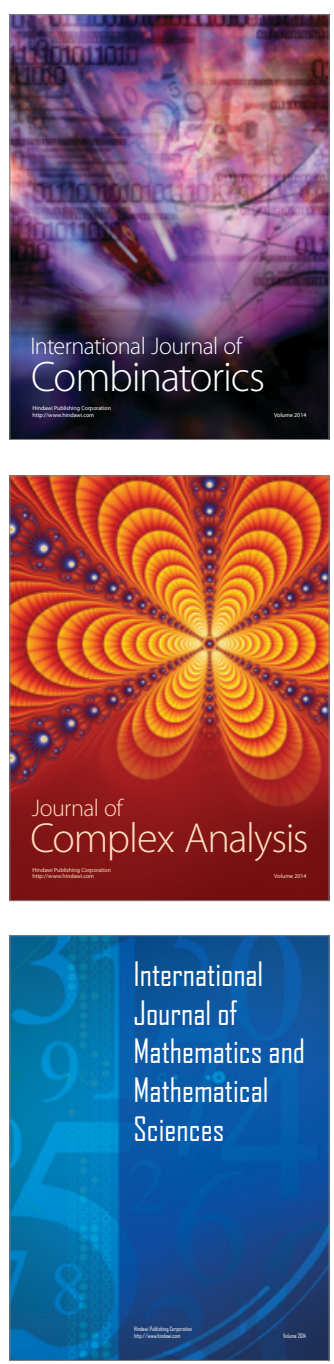
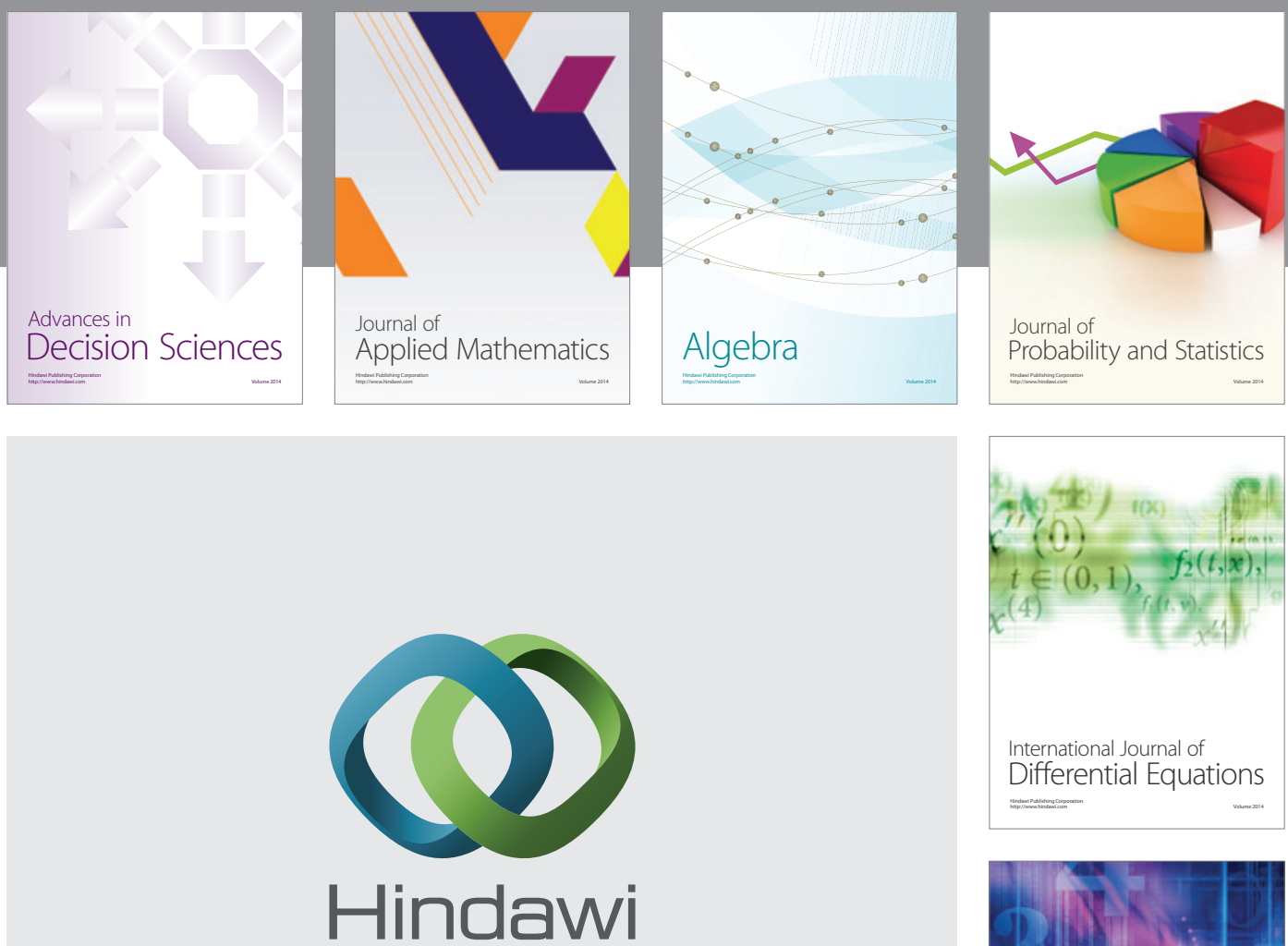

Submit your manuscripts at http://www.hindawi.com
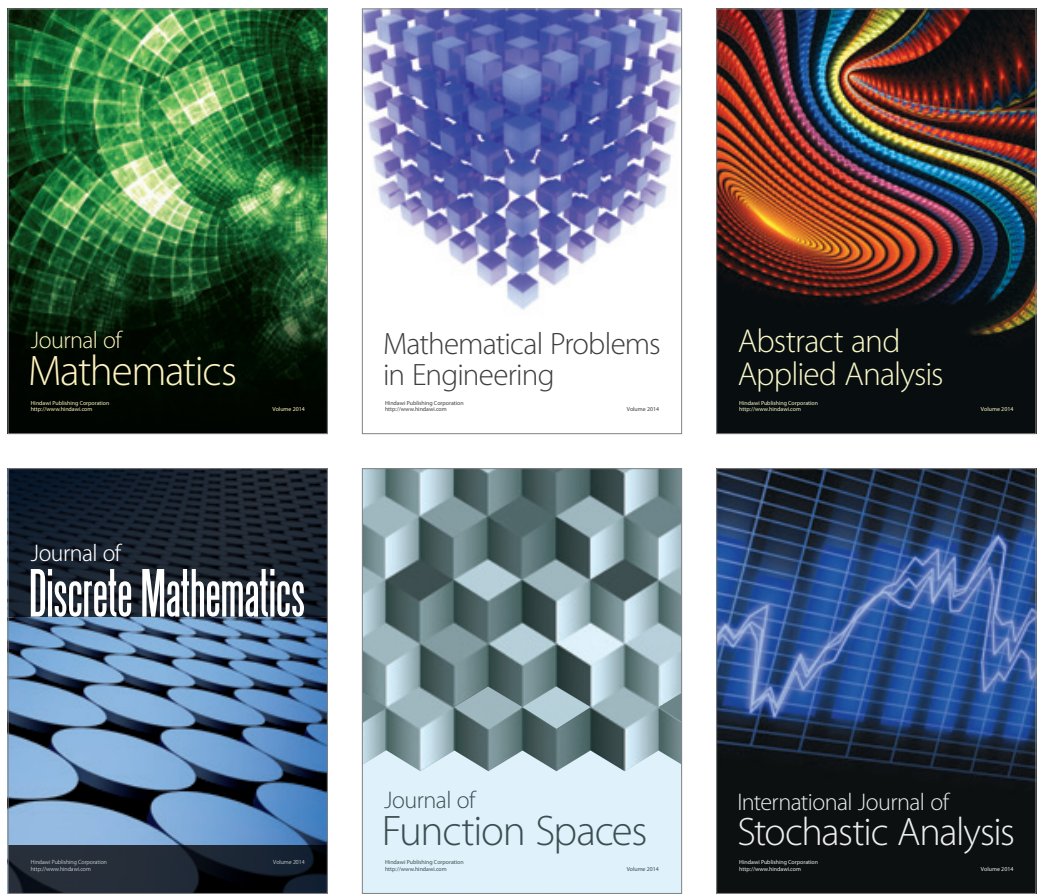

Journal of

Function Spaces

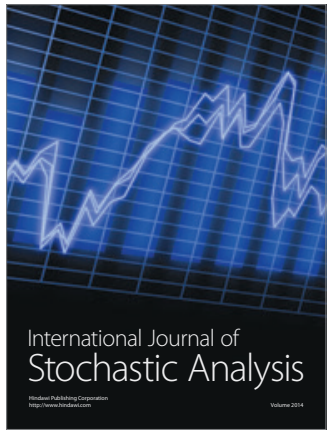

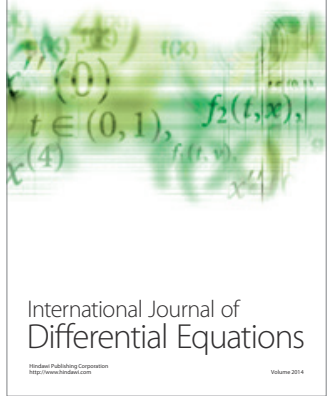
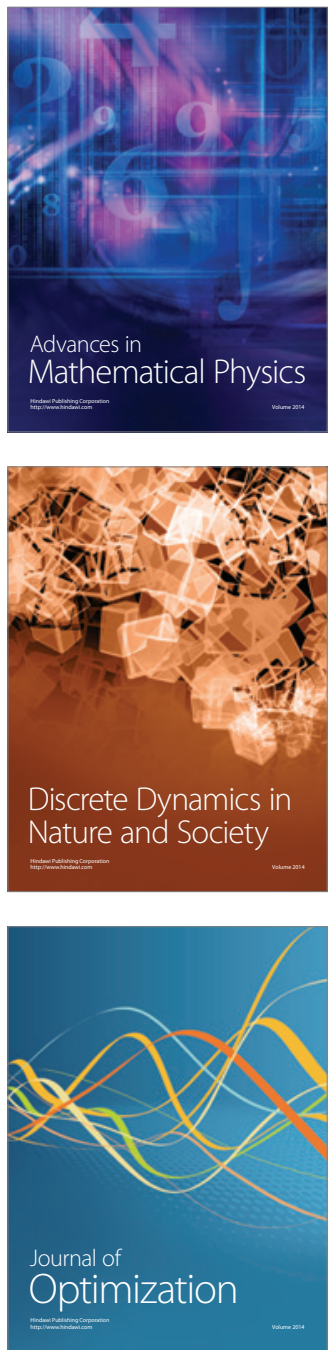